Itinéraires Itinéraires

Littérature, textes, cultures

\title{
L'espérance au conditionnel des compagnons de route (1920-1939)
}

\section{Hélène Baty-Delalande}

\section{(2) OpenEdition}

\section{Journals}

Édition électronique

URL : http://journals.openedition.org/itineraires/1422

DOI : $10.4000 /$ itineraires. 1422

ISSN : 2427-920X

Éditeur

Pléiade

\section{Édition imprimée}

Date de publication : 1 décembre 2011

Pagination : 135-151

ISBN : 978-2-296-55744-4

ISSN : $2100-1340$

Référence électronique

Hélène Baty-Delalande, «L'espérance au conditionnel des compagnons de route (1920-1939) », Itinéraires [En ligne], 2011-4 | 2011, mis en ligne le 01 décembre 2011, consulté le 19 avril 2019. URL : http://journals.openedition.org/itineraires/1422 ; DOI : 10.4000/itineraires.1422

\section{(C) $(\oplus \Theta$}

Itinéraires est mis à disposition selon les termes de la licence Creative Commons Attribution - Pas d'Utilisation Commerciale - Pas de Modification 4.0 International. 


\section{L'espérance au conditionnel des compagnons de route (1920-1939)}

\section{Abstract}

From 1920 to 1933, the few fellow travelers are fascinated by the USSR, which represents a hope for progress in the Far East, even if it remains a mythology for intellectuals refractory to the Revolution in France. From 1933 to 1939, because of the growing importance of Communist Party and the historical urgency, they become more pragmatic while dreaming of new humanism, but their real impact is questionable. (About Rolland, Guéhenno, Bloch, Gide, Malraux).

Keywords : fellow traveler, commitment, French writers, French Communist Party, Congress of Writers in Defense of Culture

Mots clés : compagnon de route, engagement, écrivains français, Parti communiste français, Congrès des écrivains pour la défense de la culture

La notion d'écrivain « compagnon de route » (poputchik) apparait en 1923 dans l'ouvrage de Trotski, Littérature et révolution. Ce dernier désigne ainsi les représentants d'un « art de transition », entre « art bourgeois » et « art nouveau ». Ni « carriéristes littéraires », ni « convertis », ils ont accepté la révolution, mais «l'idéal communiste leur est étranger »; « inquiets et instables », ils proposent un «nouveau populisme soviétique », dont les perspectives politiques demeurent inabouties. Enfin, indique Trotski, « pour un "compagnon de route", la question se pose toujours de savoir jusqu'où il suivra ${ }^{1} \gg$. Une sympathie ambiguë à l'égard de la révolution, un engagement insuffisant, un enthousiasme superficiel, des œuvres parfois irrécupérables : les ambivalences axiologiques de ce que désigne l'expression " compagnon de route ", du point de vue communiste, sont déjà présentes. Cela n'empêchera pas sa large diffusion, en France, dès les années 1924-1925. Le terme se banalise, au point qu'on le retrouve dans une acception élargie 
dans le Second manifeste du surréalisme ${ }^{2}$ (1929). Il est omniprésent en 1936, à l'heure du grand rassemblement antifasciste, dans la presse, les revues littéraires, les correspondances, et donne son titre à un ouvrage de Romain Rolland qui rassemble des essais littéraires consacrés à de grandes figures tutélaires (Shakespeare, Goethe, Gobineau, Renan, Spitteler, Hugo, Tolstoï et Lénine) ${ }^{3}$. Le « compagnon de route » intellectuel n'est pas un simple « sympathisant »; il joue à être un " camarade », rêve de fraternité, mais il n'adhère pas au Parti et reste un profane. Entre la revendication d'une foi partagée et l'insuffisance d'un engagement individuel, sa posture est fragile, parfois précieuse, souvent suspecte.

De l'insuffisance, dans la perspective communiste, à l'irresponsabilité et à l'aveuglement coupable, dans la perspective opposée : les compagnons de route ont entretenu la grande illusion meurtrière du communisme soviétique, d'autant plus efficacement qu'ils affectaient une certaine indépendance à l'égard de l'URSS et du Parti communiste. Rares étaient les contemporains qui, à gauche, criaient casse-cou, mais les condamnations rétrospectives des compagnons de route de l'entre-deux-guerres abondent, tels les travaux de David Caute ${ }^{4}$, par exemple. Les historiens tentent désormais d'échapper à cette obsession de la culpabilité et de l'aveuglement politique des intellectuels 5 . Certes, la fascination pour l'URSS et, à partir de 1932, la conviction très largement partagée que la lutte contre le fascisme passait par le rapprochement avec les Soviétiques peuvent sembler déraisonnables, sinon coupables à l'heure où l'URSS instituait un système concentrationnaire pour asseoir son pouvoir étatique. Mais il faut faire la part d'un enthousiasme souvent raisonné et de mythologies opérantes face à un dilemme tragique : le rêve de l'action, la conviction d'un rôle social de l'écrivain, la tentation intime de l'engagement, le renoncement douloureux à certaines formes d'indépendance et de sincérité, sans doute, au nom d'idéaux collectifs.

Le rapprochement d'écrivains de bonne volonté des mouvances communistes n'est pas une spécificité française; le Congrès international pour la défense de la culture de 1935 en est une preuve éclatante. S'ils

2. On lit ainsi : « À l'encontre de nos premiers compagnons de route que nous n'avons jamais songé à retenir, Desnos... » (La Révolution surréaliste, n 12, 15 décembre 1929 , p. 11).

3. Romain Rolland, Compagnons de route, essais littéraires, Paris, éd. du Sablier, 1936.

4. David Caute, Les Compagnons de route 1917-1968 [1973], Paris, Robert Laffont, 1979.

5. Michael David-Fox invite ainsi à sortir des catégories stériles de la trahison et de l'aveuglement, dans ses nombreux travaux sur les intellectuels et l'URSS (voir en particulier "The "Heroic Life" of a Friend of Stalinism : Romain Rolland and Soviet Culture ", Slavonica, vol. 11, $\mathrm{n}^{\circ}$ 1, April 2005, p. 3). Dans sa préface aux textes rassemblés par Sandra Teroni et Wolfgang Klein, Pour la défense de la culture, les textes du Congrès international des écrivains (Paris, juin 1935), Éditions universitaires de Dijon, 2005, Serge Wolikow affirme la même chose : « on peut mesurer à quel point est inadaptée la problématique des “compagnons de route", politiquement aveuglés » (p. 8). 
partagent avec les autres intellectuels européens le souvenir cuisant de la Grande Guerre et une vision ambivalente de la Russie, mêlant espérance, condescendance et clichés, les compagnons de route français sont tributaires d'une forme de romantisme révolutionnaire, hérité de l'Histoire nationale ${ }^{6}$, et cultivent une vision messianique de l'écrivain. Parallèlement, de Jaurès à Vaillant-Couturier, le Parti communiste français est particulièrement marqué par une culture intellectuelle qui résiste à la bolchevisation des années 1920, d'autant plus que les théories marxistes restent mal connues jusqu'au milieu des années 1930, et que la doctrine du Parti est alors quelque peu flottante. La politique culturelle du Parti elle-même concorde assez mal avec la ligne de Moscou, comme en témoignent les égards accordés à Barbusse jusqu'à sa mort, en 1935, malgré la condamnation de ses positions par l'URSS dès 1930. Même pendant la période de repli sectaire du PCF, de 1923 à 1934, les relations avec les écrivains sympathisants sont caractérisées par une certaine souplesse - mieux vaut être compagnon de route que dissident.

La diversité des parcours et le foisonnement des convictions ne permettent pas d'élaborer un modèle de compagnon de route à la française. À des cheminements personnels, à des drames intérieurs, parfois, se surimposent des structures, des réseaux, des lieux, au sens le plus large du terme : revues, manifestes et pétitions, congrès, voyages en URSS. De l'autre côté de la barrière, Barbusse évoque des « intellectuels en présence de la situation du monde ${ }^{7}$ ", antifascistes, anticapitalistes; une présence, donc, en deçà d'une adhésion, qui est déjà une prise de risques, un engagement aux modalités complexes, réticent et fasciné. Les espérances sont vagues, si l'ennemi est clairement identifié : le vieux monde bourgeois et ses valeurs réactionnaires, le militarisme, le capitalisme, le fascisme. La sympathie à l'égard de la révolution soviétique, et souvent de l'URSS elle-même, est une évidence; c'est l'adhésion au Parti qui ne l'est pas. Le compagnon de route veut bien être un "travailleur de l'esprit ", il rêve sans doute de « prendre place, de plus en plus consciemment, dans le grand drame collectif $^{8} »$, mais il résiste à l'enrégimentement dans le Parti.

6. Pour Jeanyves Guérin : «Le communisme, le marxisme et l'URSS sont inscrits dans le double héritage des Lumières et de la Révolution française et pensés selon leurs catégories : 1793, 1848, 1871 et 1917 forment une chaîne de dates. Ce qui se passe en URSS ne soulève aucune objection : le peuple s'y défend contre ses ennemis. Que Romain Rolland, Jean Cassou et al., qui ne sont pas membres du PCF et qui n'ont pas reçu d'éducation marxiste, en soient arrivés là, montre, si besoin était, la persistance de l'imaginaire jacobin. » (Art nouveau ou homme nouveau, Modernité et progressisme dans la littérature française $d u$ $X X^{e}$ siècle, Paris, Honoré Champion, 2002, p. 157.)

7. Lettre à Bloch du 11 novembre 1934, citée par Wolfgang Klein, Pour la défense de la culture, op. cit., p. 45.

8. Henri Barbusse, présentation de l'initiative du Congrès, Monde, no 330, 4 avril 1935, p. 3, repris dans Europe, $\mathrm{n}^{\circ}$ 149, mai 1935, p. 151-152 et dans Commune, n 20, avril 1935, p. 900-902. 
Du Congrès de Tours à la fondation de l'Association des écrivains et des artistes révolutionnaires (AÉAR), en 1932, le PCF est un parti à l'audience relativement faible; rares sont ceux qui croient encore à la possibilité d'une révolution prolétarienne en France à brève échéance depuis l'échec allemand. Peu d'intellectuels choisissent d'y adhérer, comme Barbusse qui entre au Parti en 1923, par pacifisme, lors de l'occupation de la Ruhr. Il y a bien sûr, en 1927-1928, les adhésions des surréalistes, Breton, Éluard, Péret, Aragon, Unik, et celles de quelques philosophes, Politzer, Raymond Lefèbvre, Nizan; ces adhésions entérinent une révolte absolue, elles sont essentiellement contestataires, et le malentendu ne tardera pas à être manifeste dans le cas des surréalistes. Ce n'est qu'au début des années 1930 qu'Aragon et Nizan auront la stature de grands écrivains communistes; jusque-là, l'adhésion au PCF ne concerne aucun écrivain de premier plan. Qu'il s'agisse des clartéistes, des surréalistes, de Barbusse lui-même, tous partagent une foi mystique dans la révolution, une très médiocre connaissance du marxisme et une position assez libre à l'égard des contraintes partisanes.

Entre les compromissions de Clarté et de Monde, et le désintéressement revendiqué de la $N R F$, hantée par le spectre d'une contamination, voire d'une stérilisation de l'art par le politique ${ }^{9}$, la revue Europe incarne une voie moyenne et défend l'idée d'une vocation sociale et politique de l'écrivain. On y croit à la " mission du poète ${ }^{10} »$, pour établir la fraternité entre les peuples, on y rêve d'une " patrie européenne ${ }^{11} »$. Mais la doctrine reste floue, en deçà de la revendication d'une proximité avec le communisme : c'est un regroupement « entre hommes de bonne volonté pour résister aux doctrines d'autorité, dénoncer les artifices qui prolongent l'antique oppression des esprits, signaler les chemins non frayés où s'engage la recherche humaine et définir peu à peu l'idéal nouveau ${ }^{12} »$. Après la parution de La Trahison des clercs (1927) de Benda, Europe prend activement part au débat sur la vocation de l'écrivain, à travers la prépublication des ouvrages de Berl et les chroniques de Guéhenno et Jean-Richard Bloch. Ces textes forment une constellation, entre les deux pôles formés par la mystique de l'esprit, chez Benda, et l'engagement dans l'action et dans le Parti, chez Nizan ${ }^{13}$. Berl cherche à penser le rapport nécessaire au

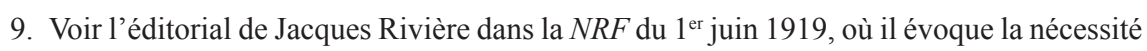
d'une revue « désintéressée » (p. 2), qui saura « nourrir à la fois, conjointes mais séparées, des opinions littéraires et des croyances politiques parfaitement définies » (p. 10).

10. Georges Duhamel, Europe, no 1, février 1923, p. 114-117.

11. René Arcos, ibid., p. 102-113.

12. Extrait de la déclaration d'intention pour la nouvelle rubrique «Chronique des idées », Europe, $\mathrm{n}^{\circ}$ 49, janvier 1927, p. 130.

13. «Il est également possible de trahir les devoirs honorables de l'esprit pour embrasser le parti terrestre des hommes. Cette nouvelle sorte de fidélité comporte la trahison à l'égard de la classe qui les asservit, des intérêts qui les écrasent » (Paul Nizan, Les Chiens de Garde [1932], Paris, Maspero, 1960, p. 133). 
politique - penser sur le communisme, en particulier, mais non penser avec : «Le clerc est gêné par la politique, non dans la mesure où il la pense, mais dans la mesure où il ne la pense pas, où il n'accomplit pas sur elle le travail qu'il accomplit sur les autres objets que l'expérience lui propose ${ }^{14}$. » De 1928 à 1932, avec Caliban parle et une série d'articles parus dans Europe, en partie repris dans Conversion à l'humain, Guéhenno tente de définir le devoir moral et politique des écrivains, sans référence directe au PCF, ni même à l'URSS. Il songe au peuple des « mangeurs de pain » et à des idées vivantes : "Les seuls engagements qui engagent sont ceux qu'on prend à l'égard des hommes et des dieux vivants, et il faut accepter d'être par eux détruit ${ }^{15}$. » Quant à Bloch, il médite sur le devenir de l'humanisme, à l'heure où le peuple devient des « masses » et où les mythes collectifs se figent en idéologies.

La correspondance échangée par Romain Rolland et Jean Guéhenno témoigne du fragile équilibre de la position de la revue à l'égard du communisme et de l'URSS. Le 20 août 1929, Rolland condamne le sectarisme du Parti communiste, incapable de condamner les injustices subies par les Géorgiens ou de reconnaître l'antifascisme de non-communistes, et proteste de son attachement aux libertés, indépendamment du Parti communiste :

\footnotetext{
Nous ne sommes qu'une poignée, dans le monde, qui ayons l'amour et le culte de la liberté - pas seulement en nous - mais chez les autres (fussent-ils nos ennemis). Cette poignée seule aurait le droit de lancer un : - « J'accuse! »-que, seul, l'avenir entendra. Je refuse ce droit aux partis de gauche ou de droite et surtout aux communistes ${ }^{16}$.
}

Mais tout au long des années 1920 et 1930, il attache une très grande attention à toute menace contre l'URSS, et en particulier aux alliances militaires qui excluent la Russie ${ }^{17}$. Progressivement, la revue devient le « compagnon de route », non pas d'un parti, mais de cette nation lointaine qui incarne le rêve de tous; il y a urgence à témoigner en faveur de l'URSS, d'abord pour sauver la paix, puis pour sauver ce modèle émergent d'une nouvelle société radieuse. Le dilemme devient de plus en plus aigu : les valeurs intellectuelles par excellence, depuis la scène inaugurale de l'affaire Dreyfus - sans cesse évoquée au tournant des années 1920 -, que sont la liberté et la vérité sont sans cesse mises à l'épreuve. Y a-t-il

14. Emmanuel Berl, «Les littérateurs et la révolution », Europe, n 73, janvier 1929, p. 48.

15. Jean Guéhenno, «Âme, ma belle âme » [Europe, n 95, novembre 1930], Conversion à l'humain, Paris, Grasset, 1931, p. 199-201.

16. Romain Rolland, L'Indépendance de l'esprit. Correspondance avec Jean Guéhenno de 1919 à 1944, Paris, Albin Michel, coll. « Cahier Romain Rolland», 1975, p. 68.

17. Voir par exemple, dans cette même correspondance, la lettre du 2 octobre 1929, p. $69-70$. 
une propagande légitime ${ }^{18}$ ? Comment esquiver le Parti dans la défense et l'illustration du communisme soviétique ${ }^{19}$ ? Certains, à la revue, suggèrent qu'il est temps de se rapprocher de ceux qui incarnent l'idée communiste en France, à l'image de Chamson : « la Révolution, sur le plan concret, suppose des liens, des fidélités envers certains hommes, avec certains groupes d'hommes contre d'autres ${ }^{20} \gg$.

Avec Anatole France - figure respectée de la gauche intellectuelle, dreyfusard, socialiste, mais sans doute d'un autre temps ${ }^{21}-$, Rolland est l'un des seuls grands écrivains français à reconnaître la révolution d'Octobre ${ }^{22}$. Il se refuse cependant à tout engagement communiste, fidèle à sa « déclaration d'indépendance de l'esprit ${ }^{23} \gg$ - en laquelle Massis avait cru voir un « bolchevisme de la pensée ${ }^{24}$ », pourtant -, malgré les vigoureux appels de Barbusse, qui publie dans Clarté, le 12 février 1921, une déclaration revendiquant sa proximité avec le PC, seule force révolutionnaire, et interpelle directement Rolland dans « L'autre moitié du devoir (à propos du rollandisme) », le 3 décembre 1921. Mais pour ce dernier, le néo-marxisme est peu conforme au véritable progrès humain, tel qu'il est appliqué en Russie; il sacrifie les plus hautes valeurs au nom d'une autre raison d'État ${ }^{25}$. À Barbusse qui le traite de « bourgeois », il rétorque que

18. Lettre de Guéhenno à Rolland du 9 février 1931 : « Je suis assiégé par des agents des Soviets, qui viennent me proposer des articles plus ou moins adroits où la propagande est évidente. [...] Faut-il publier cela, qui est si habile, trop habile? Je suis dans un affreux embarras. Faut-il laisser servir une cause à laquelle on tient par des gens douteux? » (ibid., p. 147).

19. Rolland propose le 7 août 1931, pour qu'Europe reste « la seule grande revue intellectuelle et sociale d'avant-garde, en Occident », d'y introduire un " "Courrier (mensuel) de la Révolution" (titre intérieur, in petto, qui peut être voilé d'un masque moins inquiétant) », constitué des « correspondances des divers pays où s'essaient des transformations sociales, ou des réveils des peuples qui se libèrent » et d'une « chronique de l'URSS » (ibid., p. 167). 20. Recension de Mort de la pensée bourgeoise (Emmanuel Berl, Paris, Grasset, 1929) dans Europe, n 79, juillet 1929, p. 532.

21. France a publié « Pour la Révolution russe. Appel au prolétariat », dans l'Humanité du 14 juillet 1920. Pour ses quatre-vingts ans, le quotidien publie un panégyrique remarquable : « En son âge mûr, il épousa la Démocratie, malgré les leçons de Renan, son maître, et cependant, chevaleresque, il rompit des lances pour Dame Justice. Il serait maintenant l'écuyer de la révolution, si la rude femme chevauchait un peu plus doucement. [...] Et l'escorte rouge répond au grand artiste, au grand honnête homme, par un joyeux salut : salut à l'écrivain inimitable, salut au sincère ami de Jaurès, salut à l'admirateur de Lénine! » (L'Humanité, 13 avril 1924).

22. Voir David James Fisher, Romain Rolland and the Politic of Intellectual Engagement, Berkeley, University of California Press, 1988.

23. L'Humanité, 26 juin 1919 (repris dans Clarté et L'Art libre, $1^{\text {er }}$ juillet 1919).

24. Henri Massis, « Pour un parti de l'Intelligence », Le Figaro, 19 juillet 1919.

25. Romain Rolland, «Lettre ouverte à Henri Barbusse », L'Art libre, janvier 1922, p. 1. 


\begin{abstract}
la Révolution n'est pas la propriété d'un parti. La Révolution, c'est la maison de tous ceux qui veulent une humanité plus heureuse et meilleure. Elle est donc aussi mienne. Seulement, je n'y puis vivre dans une atmosphère de coterie, comme celle que veulent nous imposer, à l'envi, « bourgeois » et communistes. [...] Car nous sommes quelques-uns qui avons cette prétention (exorbitante, à ce qu'il paraît) de rester dans la Révolution, - et d'y rester des hommes libres ${ }^{26}$.
\end{abstract}

Malgré sa fascination réelle pour le mouvement révolutionnaire et pour le monde nouveau qui naît à l'Est, il se méfie du bolchevisme, de toute armée, de tout parti - fussent-ils de la révolution. Il reste fidèle aux principes de 1919, s'adressant ainsi aux écrivains tentés par le communisme : « [L]a meilleure façon de servir la cause humaine et la Révolution même, c'est de garder l'intégrité de votre pensée libre - fût-ce contre la Révolution, si elle ne comprenait pas ce besoin vital de liberté27! »

Comment expliquer, alors, le rapprochement de plus en plus explicite de Rolland avec les communistes, alors même qu'il se tourne vers l'Inde et la pensée de la non-violence de Gandhi? Il y a d'abord des combats communs à mener contre tous les impérialismes, où l'écrivain fait entendre sa différence, comme lors de la guerre du Maroc, en $1925^{28}$; ce compagnon de route est un opportuniste, qui mesure son soutien, jusqu'en $1927^{29}$. La foi dans la vocation du peuple russe - sous l'influence de Maria Koudacheva, mais aussi de l'ami Gorki - le conduit progressivement à accepter la violence, en URSS, pour le progrès social, et même la possibilité d'un conflit « pour la défense de l'URSS ${ }^{30}$ », toujours, et à tout prix, malgré

26. Romain Rolland, « Deuxième lettre à Henri Barbusse », L'Art libre, février 1922, p. 1. 27. Ibid., p. 2.

28. L'Humanité publie le texte de Rolland sur la guerre du Maroc paru dans Clarté, le 15 juillet 1925, à l'exception de ces lignes : " Quant aux communistes, qui ne voient dans ce soulèvement des peuples que la ruine de l'impérialisme, je les avertis que les forces déchâ̂nées ne distingueront pas entre l'impérialisme et le communisme d'Europe et que sous le rouleau d'Asie le bolchevisme de Moscou, un jour, sera anéanti. »

29. Rolland écrit le 19 mars 1927 à Zweig : « Vous êtes bon, vous et Romains, de dire que les communistes font tort à la cause du droit, et qu'il vaudrait mieux que cette cause fût soutenue par le parti des honnêtes gens, raisonnables et modérés! Je le pense aussi. Mais où et quand a-t-on vu les honnêtes gens, raisonnables et modérés, prendre l'initiative d'une action dangereuse contre la violence armée? Et puisqu'ils se tairont toujours, on est bien forcés de s'allier (pour un temps, pour un but précis) avec les seuls qui osent agir et parler. » (Cité par Bernard Duchatelet dans son introduction au Voyage à Moscou, Paris, Albin Michel, coll. « Cahier Romain Rolland », 1998, p. 46.)

30. «Pour la défense de l'URSS », 19 avril 1930, Monde. Cette autre déclaration est extrêmement ferme : «Franc jeu! - Moi, j’étale le mien : si l'URSS est menacée, quels que soient ses ennemis, je me range à ses côtés » (Romain Rolland, " Europe, élargis-toi, ou meurs! » [Nouvelle revue mondiale, février 1931], Quinze ans de combat, Paris, Rieder, 1935, p. 121). Le 29 mai 1929, dans une lettre à Istrati, il explique n'être " pas membre du parti communiste », mais que « si l'URSS est attaquée, [il] la défendr[a] de toutes [ses] forces. Car elle représente, avec toutes ses erreurs (qui sont humaines, qui étaient fatales), 
ses errements. L'adhésion au Parti reste alors un choix marginal; le haut patronage de Romain Rolland est symboliquement beaucoup plus efficace, pour les deux parties. Dès 1927, il invente ce qui sera le magistère des grands compagnons de route des années 1930. Tout y est : la posture messianique, la vision tragique des souffrances du monde présent, la certitude illuminée de l'avenir meilleur, l'élection d'une cause sacrée et d'une patrie de l'humanité tout entière, cette Russie archaïque et moderne à la fois :

La Révolution russe représente le plus grand effort social, le plus puissant, le plus fécond de l'Europe moderne. [...] Si elle est écrasée, ce ne sera pas seulement le prolétariat du monde qui sera asservi, mais toute liberté sociale ou individuelle - cette sainte liberté, que le bolchevisme a sottement combattue, et qui était la meilleure alliée de la Russie affranchie ${ }^{31}$.

C'est à partir de ce moment, écrit-il en 1935, qu'il n'a plus cessé « d'être le compagnon de route de la République soviétique, et de combattre à ses côtés ${ }^{32} »$.

Nouveau croisé contre la guerre et les impérialismes, pleinement engagé dans le renouveau social, en toute "indépendance d'esprit », il adopte la figure messianique du guide éclairant le peuple ${ }^{33}$ qui fonde la gloire et la responsabilité de l'écrivain - Hugo et Zola en un seul homme, donc. Il n'y a pas de dégradation de l'art dans la politique, pour lui, qui s'est toujours exprimé à travers Jean-Christophe, puis à travers Annette et les autres figures de L'Âme enchantée, fresque de la conversion au communisme. En cela, il ignore les déchirements des autres compagnons de route; son idéal esthétique a le mérite de la transparence - mais il est d'ores et déjà dépassé.

Autre génération, autres principes. Les deux chroniqueurs importants d'Europe, Bloch et Guéhenno, ont tous deux des sympathies avouées pour les idéaux d'Octobre, mais leurs trajectoires vont s'inverser à partir de 1932 : adhésion au Parti pour le premier, éloignement progressif du communisme pour le second. Après la guerre, le fondateur de L'Effort est proche du courant syndicaliste révolutionnaire. Pacifiste convaincu, il se rallie au « rollandisme » de 1919. Il s'éloigne alors progressivement du jeune PCF

le seul bastion qui défend le monde contre plusieurs siècles de la plus abjecte, de la plus écrasante réaction » (Panaït Istrati, Vers l'autre flamme, confession pour vaincus [1929], Gallimard, coll. « Folio/Essais », 1987, p. 202-203).

31. Romain Rolland, «Lettre au Libertaire sur "La répression en Russie" » [Europe, $\mathrm{n}^{\circ}$ 58, octobre 1927], Quinze ans de combats, op. cit., p. 80.

32. Préface, ibid., p. XLVI.

33. Il écrit à Guéhenno, le 17 février 1930 : « [C]ette “immense espérance”, c'est la leur. C'est leur souffle. En aurions-nous besoin, si nous étions seuls? Je n'ai plus, personnellement, aucun désir de vie, d'avenir, ou d'au-delà. Mais ce sont eux qui ont inscrit en moi (sans désir mien) cette vie qui n'a point de fin, et l'élan de leur montée. » (Romain Rolland, L'Indépendance de l'esprit, op. cit., p. 84). 
et collabore activement à Europe ${ }^{34}$. La ferveur révolutionnaire laisse place à une intense activité critique; Bloch médite sur ce qui lui apparaît comme un échec de la révolution, loin du rêve d'une grande république fraternelle et universelle, de cette mystique héritée du dreyfusisme et du romantisme de la Révolution de 1789. Ni guide messianique, ni même compagnon de route, l'intellectuel est alors « chargé d'inventorier les cadavres de mots et d'identifier les mots nouveaux, le nouveau mythe déjà présent ${ }^{35} »$, rejetant tout dogmatisme, faisant la part des mythes et des mots - faisant le deuil d'un mythe de la révolution. Le révolutionnaire, de « candidat à l'héroïsme et à la pureté », se fond désormais dans « une discipline de masse », " une pensée de troupeau », et se dédouane de toute responsabilité grâce au Parti, « merveilleuse machine pour ne pas penser $»^{36}$. Mais la mystique née de la révolution d'Octobre demeure, entêtante - celle qu'il qualifiait en 1922 d' « optimisme du pessimisme » :

Voici venir des temps où les maîtres du monde cesseront de mettre l'homme en discorde contre lui-même; ou, au lieu de lui demander un sacrifice on lui demandera de satisfaire un besoin de son âme; où, au lieu de lui faire une obligation de haïr, on lui montrera l'amour comme la suprême raison $^{37}$.

Conscience «forgée par le socialisme ${ }^{38}$ », l'essayiste demeure en deçà des engagements de Rolland; pas encore un compagnon de route, mais un écrivain fécond et une voix acide sur les contradictions du temps.

Le directeur d'Europe, quant à lui, se fait le chantre de l'engagement des écrivains, insistant sur l'enjeu éthique d'une présence au monde revendiquée, à la fois dans les actes publics et dans les œuvres. Il fait ainsi l'éloge de la « littérature militante» :

Une telle résolution rappelle aux écrivains leurs plus vrais devoirs, en leur demandant s'ils continueront d'être les amuseurs serviles et vaniteux d'une société qui s'effondre ou s'ils rejoindront le gros de l'humanité et redeviendront au moins ses " compagnons de route ». Psychologisme, esthétisme, surréalisme, dadaïsme, notre littérature se perd par la subtilité et la futilité. Reprendra-t-elle vie aux sources profondes? Retrouvera-t-elle le sens des vrais problèmes? Et d'abord, le « non » qu'elle a à dire, aura-t-elle la force et le courage de le dire? « Je ne puis me taire, s'écriait Zola, car je

34. Voir la présentation de Jean-Richard Bloch, ou l'écriture et l'action, éd. Annie Angremy et Michel Trebitsch, Paris, Bibliothèque nationale de France, 2002.

35. Jean-Richard Bloch, Destin du siècle [1931], édité et présenté par Michel Trebitsch, Paris, PUF, coll. « Quadrige », 1996, p. 178.

36. Jean-Richard Bloch, Offrande à la politique, Paris, Rieder, 1933, respectivement p. 116, p. 119 et p. 121.

37. Jean-Richard Bloch, «Optimisme du pessimisme » [Clarté, 21 décembre 1921, p. $49-51$ et 18 janvier 1922, p. 97-104], Destin du siècle, op. cit.

38. Frédéric Lefèvre, Une heure avec..., Paris, Gallimard, 1933, p. 198. 
ne veux pas être complice. » [...] Un livre dont la traduction vient de paraître en France, montre assez ce que peut être cette littérature de propagande et qu'elle grandit encore ceux qui s'y emploient, s'appelleraient-ils Gorki ou Rolland. Eux et nous, c'est un titre excellent qui nous impose un choix. C'est le recueil de quelques-uns des articles que Gorki, au jour le jour, écrit au service de la révolution, et Roman Rolland en a écrit la préface. [...] Le livre de Gorki fait la preuve qu'un libre esprit ne cesse pas d'être libre, s'il se soumet à la discipline de la révolution ${ }^{39}$.

La défense des valeurs dreyfusardes de l'esprit est ici paradoxale, quand la liberté est autorisée par la « discipline de la révolution », et fonde une œuvre de « propagande ». De tels propos sont rares, chez Guéhenno, pour qui les vrais engagements intellectuels relèvent d'une exigence avant tout humaniste, hors de tout esprit de parti. Intéressé par la révolution, informé plus que d'autres sur les formes concrètes de sa réalisation en URSS, dans le cadre de ses recherches pour une biographie de Lénine jamais menée à bien, il se dégoûte bientôt du « fatras marxiste » et la lueur orientale, que d'aucuns célèbrent à l'envi, lui paraît émaner d'un « monde gris, sans éclat ${ }^{40}$. Idéaliste, profondément imprégné d'un socialisme jaurésien, ce pur produit de la méritocratie républicaine n'a jamais oublié ses origines populaires - mais Caliban ne parle pas pour les prolétaires, il parle au nom du peuple, et ce n'est pas la même chose. Guéhenno est donc, jusqu'en 1932, un sympathisant de l'URSS, il ouvre largement sa revue aux communistes, comme aux représentants d'autres sensibilités de la gauche intellectuelle; mais il ne pourra aller plus loin aux côtés du PCF ${ }^{41}$.

Les années 1932-1933 marquent un tournant décisif; avec la création de l'Association des écrivains et des artistes révolutionnaires et l'essor des mouvements antifascistes après les Congrès d'Amsterdam et de la salle Pleyel, le compagnonnage de route devient la forme privilégiée de l'engagement antifasciste des écrivains.

«Compagnon de route »: le terme se banalise, la posture se popularise, avec les exemples éclatants de Gide, de Malraux, de Romain Rolland, et de beaucoup d'autres, plus ou moins attendus, de Bloch ${ }^{42}$ à

39. Jean Guéhenno, « Littérature prolétarienne II », Europe, n 109, janvier 1932, p. $111-115$.

40. Notes de Guéhenno prises pour son Lénine, datant de 1927-1928, citées par Nicole Racine, "Jean Guéhenno dans la gauche intellectuelle de l'entre-deux-guerres », dans Jeanyves Guérin, Jean-Kély Paulhan et Jean-Pierre Rioux (dir.), Jean Guéhenno, guerres et paix, Villeneuve d'Ascq, Presses universitaires du Septentrion, 2009, p. 77.

41. Voir Nicole Racine, « Jean Guéhenno et le communisme (1920-1939)», dans Hommage à Jean Guéhenno à l'occasion du centième anniversaire de sa naissance, Paris, Commission de la République française pour l'Éducation, la Science et la Culture (UNESCO), édition révisée, 1993, p. 73-85.

42. Voir Michel Trebitsch, «Jean-Richard Bloch et la défense de la culture », Sociétés \& représentations, $\mathrm{n}^{\circ} 15$, décembre 2002, p. 65-76. 
Benda $^{43}$, en passant par Cassou, Dabit, Guilloux, Chamson, Chardonne, Viollis. Mais la notion reste floue : on est compagnon de route jusqu'à l'adhésion au Parti, certes, mais à quel moment n'est-on plus seulement un homme de gauche, un intellectuel attentif au marxisme ou à l'expérience soviétique? À partir de 1932, la sympathie affichée à l'égard de l'URSS et de la révolution ne suffit plus pour prétendre à ce titre; on est compagnon de route $d u$ Parti. On peut ainsi hésiter sur la position d'un Guéhenno, en qui Pascal Ory voit un compagnon de route ${ }^{44}$, mais non Nicole Racine, qui souligne son refus de toute soumission, même lointaine, même indirecte, au Parti ${ }^{45}$. En 1934, la fascination de Guéhenno à l'égard du " grand feu » oriental qui fait « tout notre espoir et toute notre joie » reste intacte, mais il est déjà singulièrement conscient des ambivalences soviétiques :

\footnotetext{
Quelques hommes désespérés par le mal du monde, mais décidés et courageux, des hommes vrais et sans illusions, qui acceptaient la ruse, la violence et l'impureté, avaient utilisé le désespoir d'un peuple, et de vive force, à coups de fusils, avaient à une dictature intéressée, secrète et honteuse, substitué une autre dictature, secrète et honteuse, pour le salut de ceux qu'ils aimaient. Depuis, ils n'ont pas cessé, selon le mot de l'un d'eux, « de se comporter sérieusement avec leurs rêves ${ }^{46}$.
}

Guéhenno est perçu comme un compagnon de route par l'URSS, qui l'invite au second Congrès des écrivains soviétiques, à l'été 1934, avec Bloch et Malraux - invitation qu'il décline, tout comme il avait refusé d'être membre de l'AÉAR : aucune compromission institutionnalisée avec le régime soviétique ou le Parti communiste. Mais il est sur toutes les tribunes, à tous les congrès, aux côtés d'Aragon et de Malraux, de 1932 à 1938. Au-delà de sa résistance à "l'esprit grégaire » et à l'idée d'une « révolution impossible ${ }^{47}$ en Occident, son pacifisme s'accommode mal d'un antifascisme reposant sur une alliance inconditionnelle avec l'URSS, tel que le défendent les compagnons de route du Parti. Homme du syncrétisme, il voit dans la révolution soviétique un nouvel avatar de l'humanisme, et non une rupture décisive ${ }^{48}$; et persiste à estimer que le socialisme

43. Benda explique alors que lorsqu'il s'agit de « défendre des principes éternels », « la mystique de gauche est recevable pour le clerc » ( «L'écrivain et le politique », $N R F, \mathrm{n}^{\circ} 256$, janvier 1935, p. 170-171).

44. Voir la préface à Annie Guéhenno et Pascal Ory (dir.), Entre le passé et l'avenir, Paris, Grasset, 1979.

45. Voir Nicole Racine, « Jean Guéhenno dans la gauche intellectuelle de l'entre-deuxguerres », op. cit., p. 73-90.

46. Jean Guéhenno, Journal d'un homme de quarante ans, Paris, Grasset, 1934, p. 226.

47. Jean Guéhenno, Dernières lumières, derniers plaisirs, Paris, Grasset, 1977, p. 20.

48. Voir le débat entre Guéhenno et Benda sur cette question, dans leurs discours au Congrès pour la défense de la culture, dans Pour la défense de la culture, op. cit. 
est « le plus grand mouvement idéaliste des temps modernes ${ }^{49}$ », que la révolution soviétique n'a en rien déclassé.

Pour l'ensemble des compagnons de route déclarés, le ralliement se fait non sans déchirement; c'est au nom d'une urgence historique que la conversion au communisme est annoncée. Choix dramatique, dans des circonstances tragiques, où s'opposent deux camps - le manichéisme domine, dans les nombreuses déclarations des compagnons de route, comme dans les hésitations des autres - et aussi choix qui engage le sens même de l'action intellectuelle : chez Gide comme chez Bloch, l'œuvre littéraire semble être empêchée par l'activisme à tout crin. Entre les compagnons de route et les communistes, il y a des lieux communs : des revues, des congrès, des manifestes divers. Commune (juillet 1933-août 1939), revue de l'AÉAR, se présente comme une « revue de combat », dénonçant les « éléments mortels de la culture et de toutes les propagandes bourgeoises » et voulant faire connaître « les éléments vivants d'une culture révolutionnaire qui se développe sur tous les plans et dont les efforts et les résultats ne sauraient être isolés de l'action du prolétariat révolutionnaire ». Elle incarne un important mouvement de fond : la constitution d'un pôle antifasciste essentiellement défensif contre les menaces allemandes et italiennes (mais aussi contre la menace fasciste en France), qui se cristallise autour d'un noyau fermement ancré à gauche. La question posée dans le premier numéro reflète un sentiment largement partagé : « communisme ou fascisme, tôt ou tard il n'y aura pas d'autre choix. Où irez-vous ${ }^{50}$ ? » Créée dans l'esprit sectaire de Kharkov, l'AÉAR est progressivement devenue un lieu de (relative) ouverture et de rassemblement, préludant ainsi à la constitution du Comité de vigilance des intellectuels antifascistes (CVIA) et au soutien massif des écrivains libéraux et de gauche au Front populaire. Dans sa préface à une brochure intitulée Ceux qui ont choisi, éditée par l'AÉAR en 1933, Vaillant-Couturier écrit que «l'AÉAR poursuit avant tout le regroupement des écrivains et artistes non conformistes qui veulent lutter aux côtés du prolétariat ». Commune va être l'instrument de l'élargissement du front culturel révolutionnaire, jusqu'au grand congrès de 1935 « Pour la défense de la culture ». À partir de septembre 1936, la ligne éditoriale s'infléchit et le sous-titre devient « Revue littéraire française pour la défense de la culture ». Si Commune est sans doute d'abord la « revue des écrivains communistes dans le Front

49. Jean Guéhenno, «Encore Nietzsche» [Europe, n 121, janvier 1933, p. 119-123], Entre le passé et l'avenir, op. cit., p. 187.

50. Gérard Servèze, « Jules Romains et le fascisme », Commune, no 1, juillet 1933, p. 275. Dans la brochure de l'AÉAR (Ceux qui ont choisi) de 1933, Guéhenno écrivait lui aussi : « Si le fascisme est advenu en Allemagne, c'est qu'on n'a peut-être pas agi à temps et je crois, pour ma part, que le devoir de tous les artistes et de tous les écrivains est de dire tout de suite de quel côté ils sont» (p. 13). 
populaire ${ }^{51} »$, elle s'ouvre largement aux compagnons de route et elle est également le creuset d'une réflexion qui déborde les dogmes du Parti sur la vocation politique et sociale de l'écrivain, en particulier sur le rapport au public et sur l'idée d'une responsabilité spécifique. Parallèlement, Europe se rapproche des communistes, particulièrement après l'éviction de Guéhenno en 1936, sous l'impulsion de Jean Cassou, compagnon de route déclaré.

Quel est le magistère réel des compagnons de route? Comme l'a montré Jean-Marie Péru, un grand échange symbolique a alors lieu entre les écrivains qui mettent leur renommée au service de la réintégration du Parti communiste dans le corps de la nation, et le Parti communiste qui renonce à toute intervention dans le champ littéraire dont il reconnaît l'autonomie $^{52}$. Pour Martin du Gard, le spectacle des amis engagés aux côtés des communistes est quelque peu pathétique : « de loin, ils ont un peu l'air de grenouilles qui sautent dans la mare les unes après les autres, sans bien savoir pourquoi; pour faire comme la voisine ${ }^{53} \gg$. Il voit ainsi dans la conversion de Gide au communisme un "scandale publicitaire » pour le Parti et un « asservissement » ${ }^{54}$ de l'esprit pour l'écrivain. De fait, c'est un événement; les confidences du Journal de 1932 font couler beaucoup d'encre et l'on glose sur les compétences idéologiques de Gide, sur la cohérence de son parcours et sur la forme de son engagement. Gide participe à l'entreprise Commune, est présent à tous les grands congrès, s'exprime à des meetings, voyage en URSS, mène des délégations : toute la panoplie du compagnon de route. Esprit humaniste, épris de justice, il choisit le camp soviétique contre celui des fascismes, non sans une certaine forme de romantisme révolutionnaire, malgré qu'il en ait. L'une des déceptions les plus cuisantes, en URSS, sera pour lui de constater le conformisme ambiant, et la reconstitution d'une petite bourgeoisie imbue d'elle-même. Son Retour de l'URSS est précédé d'un avant-propos précautionneux, d'abord publié dans Vendredi le 6 novembre 1936; rendant hommage à cette terre « en gésine », « un exemple, un guide », il se défend de toute trahison, au nom de la sincérité qu'il doit à son engagement pour le plus grand nombre, et affirme bravement : « les erreurs particulières d'un pays ne peuvent suffire à compromettre la vérité d'une cause internationale, universelle $»^{55}$.

51. Titre du premier chapitre de l'ouvrage de Wolfgang Klein, « Commune », revue pour la défense de la culture (1933-1939), Paris, CNRS Éditions, 1988.

52. Jean-Michel Péru, « Une crise du champ littéraire français. Le débat sur la "littérature prolétarienne" (1925-1935) », Actes de la Recherche en Sciences Sociales, n 89, septembre 1991, p. 47-65.

53. Lettre à Gide du 27 mars 1934, Correspondance André Gide-Roger Martin du Gard, t. I, 1913-1934, introduction par Jean Delay, Paris, Gallimard, 1968, p. 609.

54. Lettre à Gide du 3 avril 1933, ibid., p. 556.

55. André Gide, Voyage au Congo. Le retour du Tchad. Retour de l'URSS. Retouches à mon « Retour de l'URSS », Carnets d'Égypte, Paris, Gallimard, 1992, p. 408-410. 
Las, les réactions sont extrêmement violentes. Guéhenno lui-même, à contre-emploi, lui reproche de desservir la cause commune; le compagnon de route ne peut aller si loin dans la critique du système soviétique - d'autant que Gide noircira considérablement le tableau dans ses Retouches, qui constituent la première dénonciation des goulags, mais aussi des conditions de vie déplorables de l'ouvrier soviétique et des procès de Moscou. Il accuse l'URSS d'avoir trahi l'idéal de la révolution d'Octobre, et le PCF de mentir au peuple. Loin d'être « une recrue de tout repos », il préfère la vérité au Parti. Il fait même le deuil de l'espérance partagée :

L'URSS n'est pas ce que nous espérions qu'elle serait, ce qu'elle avait promis d'être, ce qu'elle s'efforce encore de paraître; elle a trahi tous nos espoirs. Si nous n'acceptons pas que ceux-ci retombent, il faut les porter ailleurs. Mais nous ne détournerons pas de toi nos regards, glorieuse et douloureuse Russie. Si d'abord tu nous servais d'exemple, à présent hélas ! tu nous montres dans quels sables une révolution peut s'enliser ${ }^{56}$.

Fin d'une illusion? C'est une libération pour Gide à l'égard d'un communisme dévoyé - mais pour les communistes, pour les compagnons de route $^{57}$, et même pour Guéhenno, c'est une erreur et une trahison.

L'autre grand homme, aux côtés du Parti, c'est encore Romain Rolland. C'est l'incarnation absolue de l'indépendance et de l'honnêteté intellectuelle d'Au-dessus de la mêlée qui compte, ici, plus que l'auteur de romans-fleuves et de pièces de théâtre embourbés dans une rhétorique qui apparaît déjà pâteuse dans les années 1930 . Il entretient sa mystique de la Russie messianique, " fille du plus brûlant espoir des peuples du monde », « incarnation vivante de nos rêves » et « réalisation la plus puissante du progrès social $»^{58}$ et met ses envolées lyriques au service d'une pensée de plus en plus orthodoxe. Son ancien camarade de l'après-guerre, Marcel Martinet, devenu un dissident, a beau jeu de dénoncer son héroïsme factice, son culte fétichiste de l'URSS et son incapacité à débusquer les impostures d'un socialisme qui n'a plus rien de populaire ${ }^{59}$. Mais Rolland est désormais lui-même un fétiche, pour le Parti et pour les compagnons de route eux-mêmes; une caution morale et une grande conscience historique, associant idéal révolutionnaire jacobin, dreyfusisme et résistance avérée à tous les impérialismes. Désormais, c'est le pragmatisme qui l'emporte; il ne publiera pas ses notes sur son propre voyage en URSS, pourtant prudentes et mesurées, à tel point qu'il y ajoutera lui aussi des « retouches » à son usage personnel, des « Notes complémentaires de 1938 » prises à la lumière des

56. Ibid., p. 505.

57. Voir par exemple la lettre de Rolland à Guéhenno du 20 décembre 1937 (Romain Rolland, L'Indépendance de l'esprit, op. cit., p. 381).

58. Romain Rolland, « Pour la défense de la paix », Vendredi, n 18, 6 mars 1936, p. 1.

59. Marcel Martinet, «1922-1935. Réponse à Romain Rolland» [La Révolution prolétarienne, $n^{\circ}$ 195, 25 janvier 1936], Agone, no 31-32, 2004, p. 273-278. 
procès de $\mathrm{Moscou}^{60}$. Le compagnon de route n'est pas aveuglé; il préfère le silence à la trahison des rêves collectifs.

Malraux, lui, est loin d'être un fétiche. Il n'est pas non plus une prise glorieuse des communistes, comme semblait l'être Gide. Après s'être battu en Indochine et avoir fondé un journal pour défendre les Annamites opprimés par l'administration coloniale dans les années 1920, il rejoint les communistes français, à la pointe des combats contre le fascisme. Il publie cinq de ses discours dans Commune, partage la tribune avec les communistes, est envoyé avec Gide auprès de Hitler pour demander la libération de Dimitrov - c'est un compagnon de route efficace et omniprésent. Le ressort de son engagement n'a rien d'une conversion idéologique; Malraux se bat avant tout pour la liberté et au nom d'un certain humanisme contre le fascisme. Il ne tente guère d'affecter une quelconque orthodoxie marxiste - son discours au Congrès des écrivains soviétiques, en août 1934, exalte la conscience et la création individuelles, avec l'affirmation fameuse : «l'art n'est pas une soumission, c'est une conquête ${ }^{61}$ ». Des Conquérants à L'Espoir, ses romans célèbrent la liberté, et Trotski ne s'y était pas trompé à la lecture de La Condition humaine :

Les sympathies, d'ailleurs actives, de l'auteur pour la Chine insurgée sont indiscutables. Mais elles sont corrodées par les outrances de l'individualisme et du caprice esthétique. [...] Une bonne inoculation de marxisme aurait pu préserver l'auteur des fatales méprises de cet ordre ${ }^{62}$.

Son exaltation bien réelle de la révolution répond au refus d'un individualisme sclérosant; le marxisme lui apparaît comme un mythe collectif commode, qui a fait naître une nouvelle forme de fraternité entre les communistes combattants. Reste que l'engagement auprès de Moscou et du Parti communiste est ferme, comme en témoigne cette déclaration de 1933 : «Quel que soit notre désir d'unité ouvrière, nous saurons toujours trouver ceux qui servent véritablement le prolétariat; en cas de guerre, même si la Russie n'y est pas engagée, nous nous tournerons par la pensée vers Moscou, nous nous tournerons vers l'Armée rouge ${ }^{63}$. $\gg$ Malraux est tout à fait fasciné par le drame, l'héroïsme, la " réalité tragique », non seulement de la révolution passée, mais du quotidien soviétique; il est fasciné par Trotski, ce héros humilié ${ }^{64}$, par la « force primitive » de la vie

60. Romain Rolland, Voyage à Moscou, juin-juillet 1935, édition et présentation de Bernard Duchatelet, Paris, Albin Michel, coll. « Cahier Romain Rolland », 1992.

61. Discours publié dans Commune, septembre-octobre 1934, repris dans La Politique, la culture, discours, articles, entretiens, présentés par Janine Mossuz-Lavau, Gallimard, coll. « folio », 1996, p. 106.

62. Léon Trotski, La Révolution permanente, Paris, Gallimard, 1963, p. 252-253.

63. Discours du 21 mars 1933 à la réunion organisée à Paris par l'AÉAR, La Politique, la culture, op. cit., p. 78.

64. Voir André Malraux, « Trotsky », Marianne, 25 avril 1934, p. 3. 
en URSS. Son rêve de communisme soviétique est le rêve d'un mythe ou d'un grand roman de la « fraternité virile ${ }^{65} »$. L'expérience de la guerre d'Espagne entame le mythe et Malraux s'éloigne des communistes; Commune ne recense pas L'Espoir. Fin du compagnonnage, en toute discrétion.

Procès de Moscou, informations chiffrées sur la misère du peuple soviétique, camps de concentration, et finalement pacte germano-soviétique : la fidélité des compagnons de route est soumise à rude épreuve. À lire Guéhenno, l'on mesure le drame intime de ces engagements collectifs, contre le fascisme, pour un idéal vague et d'autant plus cher. Alors qu'Europe se tait sur les procès de Moscou, il écrit dans Vendredi, le 16 octobre 1936 : « Nous nous sommes retenus de prendre parti tout de suite sur une si grave question quand les passions étaient surexcitées et dans un instant où tous nos adversaires exploitaient contre des camarades, contre le parti communiste français, un tel événement. » Le 5 février 1937, il précise sa position : « À propos de ce procès de Moscou, je voudrais ne rien écrire qui pût décourager ou attrister ceux-là de nos camarades pour qui Moscou sera toujours le cœur du monde. La Russie leur semble sainte et on comprend du reste leur piété et leur amour. " Pendant ce temps, Bloch adhère au Parti et Europe est toujours davantage inféodée aux communistes. Fin (provisoire) de l'aventure des compagnons de route, le pacte germano-soviétique brise cette idée essentielle que la lutte contre le fascisme ne peut triompher que si elle est aussi une défense de l’URSS.

Revenant sur cette grande époque des compagnons de route, Malraux écrit que « les Fronts populaires d'Espagne et de France furent à la fois la conséquence d'une analyse marxiste, et la revanche de Michelet sur Marx. L'éclat d'Europe naquit de cette coexistence, qui s'appelle fraternité66 ». Le Congrès pour la Défense de la culture de 1935 marque bien une tentative collective, en particulier chez les compagnons de route français, de résoudre un paradoxe, entre Marx et Michelet. Les années 1920 sont celles de la mystique d'Octobre, chez les premiers compagnons de route; à l'image de Rolland, ils cultivent une imagerie révolutionnaire, rêvent d'une Russie exemplaire mais qui reste à bien des égards exotique - la Révolution russe est spécifiquement russe et n'est pas transposable dans la civilisation occidentale. L'universel est donc éminemment particulier, et Guéhenno 1'exprime sans ambages :

Il y avait assez de lumière de France pour que nous continuions à ne compter que sur la raison. La Révolution ne peut s'y faire contre la liberté. Le génie de Lénine avait été d'opposer à une autocratie policière une

65. Discours de compte rendu du Congrès des écrivains soviétiques, 23 octobre 1934, paru dans Monde et Commune, repris dans La Politique, la culture, op. cit., p. 109-117. 66. Introduction à Romain Rolland, L'Indépendance de l'esprit, op. cit., p. 9. 
révolution policière. Nous n'avions nul besoin, quant à nous, d'une Tcheka ou d'un Guépéou ${ }^{67}$.

La lueur à l'Est enthousiasme, émeut - mais de là à se rallier au PCF, et à accepter la bolchevisation de la patrie de l'indépendance de l'esprit, non. Les compagnons de route voient la Révolution russe à travers le prisme de leurs propres mythes révolutionnaires, jacobins, soréliens, simplement socialistes; mais la fascination est aussi traversée par un sentiment de profonde étrangeté.

Les années 1930 ne voient pas la réduction de ce sentiment d'étrangeté - les récits de voyage en URSS en portent témoignage ${ }^{68}$. Mais l'urgence d'un combat antifasciste qui n'a rien d'exotique et le rapprochement stratégique et affectif avec les communistes permettent de réduire cette contradiction originelle entre une foi collective sincère et l'impossible universalité de la révolution soviétique. Reléguant à l'arrière-plan l'idéal révolutionnaire, les compagnons de route fondent leurs engagements sur la notion de culture et sur une conception élargie de l'humanisme - version œcuménique d'un idéal fraternel, populaire et progressiste. Les rassemblements divers des années 1930 rationalisent des affects, à l'heure du drame collectif; le sentiment très vif d'un idéal commun, aussi vague soit-il, conforte la proximité objective des compagnons de route et des communistes.

Ces engagements des compagnons de route n'ont sans doute pas fondamentalement orienté la production littéraire de l'entre-deux-guerres, mais ils ont nourri une inquiétude lancinante sur la posture de l'écrivain et la valeur de son œuvre. Les débats portent d'abord sur la morale de l'écriture, contre tout enrôlement des esprits; les compagnons de route écrivent pour le peuple, mais les réflexions sur la littérature prolétarienne ou le populisme, par exemple, restent marginales; il n'y a pas encore de véritable politique littéraire au Parti. Après 1933, la question des rapports entre littérature et politique devient tout à fait centrale, à l'heure des activismes. L'écrivain compagnon de route est un porte-conscience, selon la formule de Bloch au Congrès de 1935; il se défend de tout asservissement idéologique, mais est pénétré de l'idée de sa propre responsabilité à l'égard du peuple et du mouvement de l'Histoire et s'engage hors l'œuvre, dans l'œuvre, pour son temps.

Hélène Baty-Delalande Université Paris 7 - CERILAC

67. Jean Guéhenno, Dernières lumières, derniers plaisirs, op. cit., p. 19.

68. Voir Michael David-Fox, "The Fellow Travelers Revisited. The "Cultured West" Through Soviet Eyes ", Journal of Modern History, n 7, 2003, p. 300-335; Sophie Cœuré, La Grande lueur à l'Est. Les Français et l'Union soviétique 1917-1939, Paris, Seuil, 1999; et Rachel Mazuy, Croire plutôt que voir. Voyages en Russie soviétique 1919-1939, Paris, Odile Jacob, 2002. 